\title{
The clinical course and diagnostic relevance of olfactory loss in a SARS-CoV-2 infection*
}

\section{M.J. Bauwens, S. Claeys}

Department of Head and Skin, Faculty of Medicine and Health Sciences, University Hospital Ghent, Belgium
Rhinology Online, Vol 4: $17-23,2021$

http://doi.org/10.4193/RHINOL/20.081

*Received for publication:

November 1, 2020

Accepted: January 19, 2020

Published: February 18, 2021

\begin{abstract}
Background: The acute onset of olfactory and/or gustatory dysfunction is a frequent complaint during the COVID-19 pandemic. It is predominantly reported by subjects with asymptomatic to mild disease severity during the early stage of the infection. The high prevalence of anosmia/dysosmia and/or ageusia/dysgeusia in this current era implements a strong correlation with a SARSCoV-2 infection and these symptoms could therefore be seen as important prodromes. The purpose of this study was to outline the occurrence, epidemiology and clinical course of olfactory and/or gustatory disorders in (suspected) COVID-19 cases and to analyse the diagnostic significance of these neurosensory dysfunctions.
\end{abstract}

Methods: An online questionnaire was carried out which addressed 500 participants with new onset olfactory and/or gustatory impairment during the COVID-19 pandemic.

Results: Acute olfactory and gustatory loss was reported by 487 (97.4\%) and 464 (92.8\%), respectively. A significant higher prevalence of neurosensory complaints was reported by women and people of younger age. The most prevalent concurrent symptoms were fatigue, headaches, nasal congestion, dry cough, rhinorrhoea and sneezing. The recovery rate after 8 weeks was $41.9 \%$ for olfactory impairment and $53.7 \%$ for gustatory impairment. Among the 93 subjects tested, $82(88.2 \%)$ tested positive for SARSCoV-2.

Conclusion: Olfactory and/or gustatory disorders are prevalent clinical findings during the COVID-19 pandemic. Neurosensory impairments, isolated or in association with other mild complaints, need to be addressed as potential symptoms of a SARS-CoV-2 infection and should be implemented as clinical markers.

Key words: COVID-19, gustatory loss, neurosensory dysfunctions, olfactory loss, SARS-Cov-2

\section{Introduction}

Since the outbreak of the Severe Acute Respiratory Syndrome Corona Virus 2 (SARS-CoV-2) in Wuhan City in early December 2019, a global expansion of the virus has occurred which resulted in a worldwide distribution of the COVID-19 disease ${ }^{(1)}$. The disease has a broad spectrum of clinical presentations and is associated with high morbidity and mortality rates ${ }^{(2)}$. The most common symptoms reported by patients are fever, dyspnoea, retrosternal pain, dry cough, sore throat, nasal congestion or rhinorrhoea, sneezing, fatigue, myalgia, malaise, headache, nausea or vomiting and diarrhoea ${ }^{(3)}$. Within the context of this current pandemic, it has been noticed worldwide that an infection with the SARS-CoV-2 virus can be accompanied by olfactory or gustatory impairment ${ }^{(4-6)}$. The olfactory complaints are predominantly present in younger patients without obvious respiratory complaints ${ }^{(7,8)}$. The absence of common symptoms (fever, cough, myalgia, headache) in this population of potentially infected patients probably results in an incorrect estimation of the presence of a SARS-CoV-2 infection, which can lead to imprudent behaviour and a reduced adherence to the imposed 
measures ${ }^{(8)}$.

Post-viral olfactory dysfunction is a known consequence of respiratory tract infections caused by a wide range of pathogens (e.g. rhinovirus, influenza virus, parainfluenza, respiratory syncytial virus, adenovirus) and covers $40 \%$ of the causes of reported anosmia ${ }^{(9,10)}$. The significant increase of reported disturbed smell and taste during this COVID-19 crisis might imply a correlation between olfactory dysfunction and infection by this specific coronavirus ${ }^{(4,11,12)}$. The aim of this research is to elaborate the presentation of olfactory dysfunction in a SARS-CoV-2 infection and to outline its epidemiology.

\section{Materials and methods}

\section{Study design and population}

The population of this study consists of 500 participants between 18 and 65 years old who experienced sudden olfactory and/or gustatory loss during the SARS-COV-2 pandemic. This retrospective study was performed by means of an online survey which was distributed via social media (Facebook, WhatsApp, etc.) during April 2020. The survey was available in three languages (Dutch, English, French) and was predominantly distributed in Belgium and neighbouring countries. All participants were asked to describe the course of their complaints and a possible link with COVID-19 was explored. Incomplete questionnaires (progress rate $<94 \%$ ) were excluded from the analysis, which resulted in 500 representative responses. All other surveys were used for the analysis. If a subject did not wish to answer the question, it was marked as a non-response. An informed consent was presented to the participants at the initiation of the survey. The study protocol was approved by The Ethic Committee of Ghent University Hospital.

\section{Statistical analysis}

Statistical analysis was performed using SPSS, version 25 (IBM). All tests were two-tailed, and a level of $p<0.05$ was used to determine statistical significance. Data-cleaning was conducted to ensure that the data did not contain aberrant values. If the respondent chose not to respond to the question, the response was replaced by a missing value. Normality of all continuous variables was verified through the range of skewness and kurtosis (between -2 and 2), as well as the visual assessment of the histogram and QQ-plot.

\section{Results}

Demographic and epidemiological characteristics A total of 500 patients were included in the study. The mean age of the population was $29.82 \pm 0.55$ years (range 18-65 years), and gender distribution was 148 (29.8\%) males versus 351 (70.2\%) females. The study population mainly originated from Belgium: 273 (54.0\%) participants were Belgian citizens,
Table 1. Demographic and epidemiological characteristics.

\begin{tabular}{|c|c|c|c|}
\hline & & $\mathbf{N}$ & $\%$ \\
\hline & hean (SD) & $29.82 \pm 0.55$ & \\
\hline \multicolumn{4}{|c|}{ Sex } \\
\hline- & M & 148 & 29.8 \\
\hline- & $\mathrm{F}$ & 351 & 70.2 \\
\hline \multicolumn{4}{|c|}{ Language } \\
\hline- & Dutch & 384 & 76.8 \\
\hline- & English & 25 & 5.0 \\
\hline- & French & 91 & 18.2 \\
\hline \multicolumn{4}{|c|}{ Country } \\
\hline- & Belgium & 273 & 54.6 \\
\hline- & France & 2 & 0.4 \\
\hline- & The Netherlands & 3 & 0.6 \\
\hline- & United Kingdom & 2 & 0.4 \\
\hline- & Unknown & 220 & 44.0 \\
\hline \multicolumn{4}{|c|}{ Tobacco use } \\
\hline- & Smoker & 77 & 15.4 \\
\hline & Daily & 23 & 4.6 \\
\hline & Weekly & 15 & 3.0 \\
\hline & o Monthly & 21 & 4.2 \\
\hline & Yearly & 18 & 3.6 \\
\hline- & Non-smoker & 400 & 80.0 \\
\hline- & Ex-smoker & 23 & 4.6 \\
\hline \multicolumn{4}{|c|}{ Alcohol use } \\
\hline- & Yes & 360 & 72.0 \\
\hline- & No & 32 & 6.4 \\
\hline- & Missing & 108 & 21.6 \\
\hline & atients & 143 & 28.6 \\
\hline \multicolumn{4}{|c|}{ Comorbidities } \\
\hline- & No comorbidities & 455 & 91.0 \\
\hline- & Diabetes mellitus & 4 & 0.8 \\
\hline- & Cardiovascular diseases & 4 & 0.8 \\
\hline- & Lung diseases & 7 & 1.4 \\
\hline- & Kidney disease & 2 & 0.4 \\
\hline- & Immunosuppression & 12 & 2.5 \\
\hline
\end{tabular}

while respectively $2(0.4 \%), 3(0.6 \%)$ and $2(0.4 \%)$ participants originated from France, The Netherlands and the United Kingdom. Smoking was reported by 77 (4.2\%) individuals with a mean number of cigarettes of $13.09 \pm 2.06$ a day. Alcohol use was present in 360 (72.0\%) participants with a weekly mean of $5.88 \pm 0.32$ units per day. Allergies were reported by 143 (28.6\%) individuals (Table 1).

Clinical course of the olfactory/gustatory dysfunction and associated complaints

Olfactory complaints were reported by 487 (97.4\%) of the participants in this study. These complaints consist of a distortion of sense of smell in 16 participants (3.2\%), a reduced sense of smell in 67 participants (13.4\%) and a complete absence of sense of smell in 404 participants (80.8\%). After 8 weeks, 200 individuals $(40.8 \%)$ reported to have recovered from their disturbance. Gustatory complaints were reported by 464 (92.8\%) individuals. These complaints consist of a distortion of sense of taste in 23 participants (4.6), a reduced sense of taste in 154 participants (30.8\%) and a complete absence of sense of taste in 287 parti- 
Table 2. Olfactory and gustatory complaints.

\begin{tabular}{|c|c|c|c|c|c|c|}
\hline & $\mathbf{N}$ & $\%$ & $\begin{array}{l}\text { Mean duration } \\
\text { (weeks) }\end{array}$ & $\begin{array}{l}\text { Median duration } \\
\text { (weeks) }\end{array}$ & $\begin{array}{c}\text { Range } \\
\text { (weeks) }\end{array}$ & $\begin{array}{l}\text { Recovery rate } \\
\text { after } 8 \text { weeks }\end{array}$ \\
\hline $\begin{array}{ll}\text { Olfactory complaints } \\
- & \text { Parosmia } \\
- & \text { Hyposmia } \\
- & \text { Anosmia }\end{array}$ & $\begin{array}{c}487 \\
16 \\
67 \\
404\end{array}$ & $\begin{array}{c}97.4 \\
3.2 \\
13.4 \\
80.8\end{array}$ & 3.97 & 4 & $0-8$ & $204 / 487$ (41.9\%) \\
\hline $\begin{array}{ll}\text { Gustatory complaints } \\
- & \text { Parageusia } \\
- & \text { Hypogeusia } \\
- & \text { Ageusia }\end{array}$ & $\begin{array}{c}464 \\
23 \\
154 \\
287\end{array}$ & $\begin{array}{c}92.8 \\
4.6 \\
30.8 \\
57.4\end{array}$ & 3.48 & 3 & $0-8$ & $249 / 464$ (53.7\%) \\
\hline
\end{tabular}

cipants (57.4\%). After 8 weeks, 249 individuals (49.8\%) experienced a recovery of their gustatory function (Table 2 , Figure $1 \mathrm{a}$ and $1 \mathrm{~b})$.

Among the 500 participants, 36 (7.4\%) reported to experience isolated olfactory complaints while 13 (2.6\%) claimed to experience isolated gustatory complaints. Concurrent olfactory and gustatory complaints were indicated by 451 (92.6\%) individuals. The statistical analysis showed a marginal significant difference ( $p=0.067$ ) between the occurrence of dysosmia in both sexes. A significant difference $(p=0.012)$ was found for the mean age of people with or without olfactory complaints. The mean age of people with olfactory complaints (29.60 y) was significantly lower than the mean age of those without olfactory complaints (38.15 y). No significant association was found between the olfactory dysfunction and tobacco use. The association between the olfactory complaints and seasonal allergy was not significant.

The most prevalent general symptoms associated with the olfactory or gustatory loss were fatigue (79.4\%), headaches (55.8\%), nasal congestion (46.6\%), dry cough (44.4\%), rhinorrhoea (41.4\%) and sneezing (40.8\%). Other symptoms that were reported by the individuals were myalgia (38.8\%), sore throat $(30.8 \%)$, sputum (27.6\%), fever (27.2\%), dyspnoea (27.2\%), retrosternal pain (21.6\%), mucous cough (18.8\%), diarrhoea (16.0\%), nausea (11.2\%), earache (11.0\%) and vomiting (2.0\%). No other complaints were reported by $8.0 \%$ of the participants. In these cases, olfactory of gustatory loss was reported as an isolated symptom
(Table 3, Figure 2).

A difference in duration was observed between the olfactory or gustatory complaints (17.95 \pm 0.529 days) and the associated symptoms. A significant difference in the mean duration was found for nasal congestion ( $p<0.001)$, nasal run $(p<0.001)$, dry cough ( $p<0.001)$, mucous cough $(p<0.001)$, sore throat $(p<$ $0.001)$, sneezing $(p<0.001)$, earache $(p<0.001)$, headache $(p<$ $0.001)$, fatigue $(p=0.024)$, myalgia $(p<0.001)$, fever $(p<0.001)$, dyspnoea $(p=0.003)$, retrosternal pain $(p<0.001)$, nausea $(p<$ $0.001)$ and diarrhoea $(p=0.001)$.

\section{Perception}

The most frequent feeling that individuals with sudden onset of olfactory or gustatory loss reported was hindrance $(n=374$; $74.9 \%$ ). Other sentiments that were declared by the participants in this study were anger $(n=197,39.4 \%)$, insecurity $(n=189$, $37.9 \%)$, depression ( $n=174,34.9 \%)$ and frustration $(n=386$, 7.2\%). Participants mainly experienced hindrance due to their olfactory or gustatory loss during the following activities: eating ( $n=468 ; 93.6 \%)$, cooking ( $n=369,73.8 \%)$, assessment of food freshness ( $n=279 ; 55.8 \%)$, assessment of personal hygiene ( $n$ $=245 ; 49.0 \%)$, detection of danger $(n=143 ; 28.6 \%)$, domestic work $(n=77 ; 15.4 \%)$, social activities $(n=50 ; 10.0 \%)$ and the practice of their occupation.

\section{Management}

362 (72.4\%) out of 500 individuals did not seek any external help
A

$$
\text { Olfactory complaints }
$$

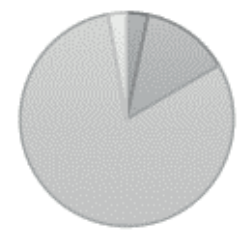

$\square$ Parosmia $\square$ Hyposmia $\square$ Anosmia $\square$ No olfactory complaints
B

\section{Gustatory complaints}

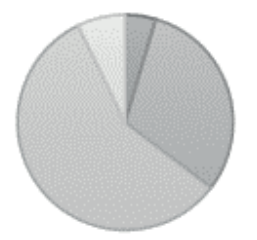

口Parageusia $\square$ Hypogeusia $\square$ Ageusia $\square$ Nogustatory complaints

Figure 1. A) Olfactory complaints and B) gustatory complaints. 


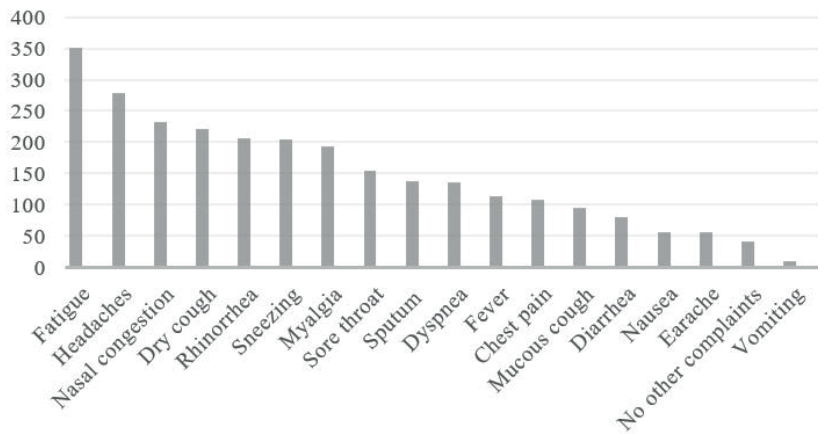

Figure 2. Associated complaints.

to resolve their olfactory and gustatory dysfunction. Among those who took medical advice, 90 (18.0\%) individuals consulted a general practitioner, 25 (5.0\%) went to the pharmacist, 15 (3.0\%) consulted an ENT physician and 22 (4.4\%) searched other forms of assistance. The initiated treatments with the purpose of restoring the olfactory and gustatory function are shown in Figure 3. A success rate was calculated based on the outcome of the initiated treatment and represents the subjective improvement noticed by the participants under the therapy. The recovery that the subjects who attempted a treatment obtained where as follows: olfactory training (95\%), nasal saline irrigations (93.0\%), essential oils (85.7\%), nasal corticosteroids (66.7\%) and nasal decongestants (65.2\%), alfa lipoic acid (50.0\%), oral corticosteroids (40.0\%) and zinc sulphate (16.7\%). Spontaneous improvement can evidently be an important confounding factor.

\section{Association with COVID-19}

Among the 500 participants in this study, 34 (7.0\%) assumed not to have interacted with a SARS-CoV-2 infected individual before the onset of their olfactory and/or gustatory complaints. Contact with a suspected case of COVID-19 was reported by 118 (24.3\%) individuals while 144 (29.6\%) stated that they had been in touch with a COVID-19 confirmed case. The remaining 190 (39.1\%) participants did not know if they had been into contact with a SARS-COV-2 infected person. No response was given to this question by 14 (2.8\%) of the participants.

Among the individuals with an olfactory and/or gustatory dysfunction, 75 (15.4\%) were not under the assumption that they were infected with SARS-COV-2. 255 (52.5\%) subjectively suspected to be infected with SARS-COV-2. The diagnosis of COVID-19 was made by a physician for 153 (31.5\%) participants. $3(0.6 \%)$ individuals could not tell if they were potentially infected. No response was given to this question by 14 (2.8\%) of the participants.

The majority, 393 (80.9\%) out of the 500 subjects, did not get a SARS-COV-2 test. A diagnostic test was performed among 93
Table 3. Associated complaints.

\begin{tabular}{|c|c|c|c|c|}
\hline \multirow[t]{2}{*}{ Associated symptoms } & \multirow[t]{2}{*}{$\mathbf{N}$} & \multirow[t]{2}{*}{$\%$} & \multicolumn{2}{|c|}{ Duration (days) } \\
\hline & & & Mean \pm SD & Median \\
\hline \multicolumn{5}{|l|}{ ENT-symptoms } \\
\hline Nasal congestion & 233 & $46.6 \%$ & $13.11 \pm .768$ & 9.00 \\
\hline Rhinorrhea & 207 & $41.4 \%$ & $12.57 \pm .773$ & 9.00 \\
\hline Sore throat & 154 & $30.8 \%$ & $9.70 \pm .762$ & 6.00 \\
\hline Sputum & 138 & $27.6 \%$ & $15.99 \pm 1.127$ & 13.50 \\
\hline Sneezing & 204 & $40.8 \%$ & $11.91 \pm .722$ & 8.00 \\
\hline Earache & 55 & $11.0 \%$ & $11.20 \pm 1.637$ & 7.00 \\
\hline \multicolumn{5}{|l|}{ Other symptoms } \\
\hline Fatigue & 352 & $79.4 \%$ & $16.39 \pm 687$ & 14.00 \\
\hline Headaches & 279 & $55.8 \%$ & $11.71 \pm .734$ & 7.00 \\
\hline Dry cough & 222 & $44.4 \%$ & $13.77 \pm .719$ & 10.00 \\
\hline Mucous cough & 94 & $18.8 \%$ & $12.97 \pm 1.085$ & 10.00 \\
\hline Fever & 114 & $27.2 \%$ & $9.10 \pm .983$ & 5.00 \\
\hline Myalgia & 194 & $38.8 \%$ & $10.53 \pm .810$ & 6.00 \\
\hline Dyspnea & 136 & $27.2 \%$ & $14.79 \pm 1.029$ & 10.50 \\
\hline Retrosternal pain & 108 & $21.6 \%$ & $11.80 \pm 1.050$ & 7.00 \\
\hline Nausea & 56 & $11.2 \%$ & $11.00 \pm 1.420$ & 7.00 \\
\hline Vomiting & 10 & $2.0 \%$ & $15.70 \pm 5.560$ & 10.00 \\
\hline Diarrhea & 80 & $16.0 \%$ & $12.25 \pm 1.664$ & 6.50 \\
\hline No other complaints & 40 & $8.0 \%$ & & \\
\hline
\end{tabular}

(18.6\%) participants, 11 (2.3\%) got a negative result while 82 (16.9\%) tested positive for SARS-COV-2. No response was given to this question by 14 (2.8\%) of the participants. Out of the 93 subjects who were tested, 82 (88.2\%) obtained a positive test result for SARS-CoV-2.

Only 7 (1.4\%) out of 500 participants were admitted at the hospital in the context of a SARS-CoV-2 infection. No hospitalization was reported by 479 (98.6\%) individuals. No response was given to this question by 14 (2.8\%) of the participants.

Among the 500 participants, 455 (91.0\%) were not known with any comorbidities whilst respectively 4 (0.8\%), 4 (0.8\%), 7 (1.4\%), $2(0,4 \%)$ and 12 (2.5\%) suffered from Diabetes Mellitus type 2, cardiovascular disease, lung disease, kidney disease and immunosuppression.

A multiple linear regression was used to predict the occurrence of olfactory impairment based on gender, age, smoking behaviour, seasonal allergy, potential contact with a SARS-CoV-2 infected subject, suspected infection with SARS-COV-2 and a positive test result for SARS-CoV-2. A significant regression was found for gender $(\beta=0.039 ; p=0.024)$, age $(\beta=-0.001 ; p=0.047)$ and $a$ suspected infection with SARS-COV-2 $(\beta=0.077 ; p=0.004)$. 


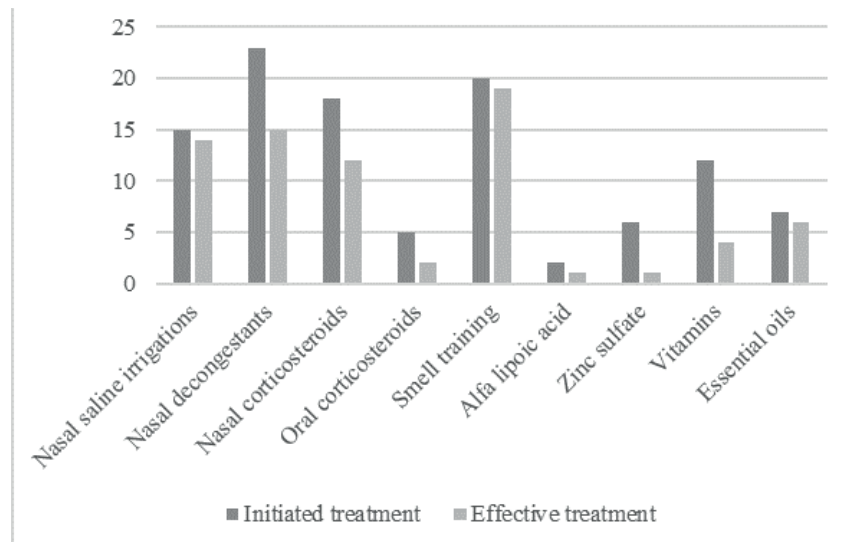

Figure 3. Treatment.

\section{Discussion}

The purpose of this research was to investigate the occurrence, presentation and diagnostic significance of olfactory and/or gustatory disorders in (suspected) COVID-19 cases. Acute olfactory and gustatory complaints were reported by respectively $97.4 \%$ and $92.8 \%$ of the participating subjects, who indicated a high prevalence of neurosensory dysfunctions during the COVID-19 pandemic. This outcome supports previous research regarding this matter which stated that neurosensory complaints are an important manifestation of an infection with SARS-CoV-2 ${ }^{(1,12)}$.

The recovery rate after 8 weeks from an olfactory dysfunction was $40.8 \%$, whilst the rate for people with gustatory loss was $49.8 \%$. A lot of research is currently being done to investigate the underlying mechanism, progression and recuperation of neurosensory impairment in a SARS-CoV-2 infection. However, the exact pathogenesis of this phenomenon has not yet been clarified. Post-viral damage to the structures of the olfactory system, more specific the neuro-epithelial cells, is observed in patients infected by this specific virus ${ }^{(13)}$. These results provide further support for the hypothesis that the olfactory loss caused by this virus can be of post-viral origin, in accordance with postviral neurosensory impairment caused by other viruses (Influenza, Rhinovirus and SARS-CoV) ${ }^{(14)}$. In these cases, spontaneous recovery is seen in $32-66 \%$ of the patients and can occur until 2 years $\left({ }^{2}, 14\right)$. Therefore, a prolonged follow-up of the recuperation will be necessary to further investigate the recovery rates over a longer period of time.

A significant part (i.e. 92.6\%) of the study population indicated to experience concurrent olfactory and gustatory complaints. From this observation, it can be concluded that there is a strong association between both disorders. Gustatory loss is rarely an isolated complaint and is in most cases observed as a manifestation of olfactory impairment because of the strong collaboration of the olfactory and gustatory functions ${ }^{(15)}$. Consequently, a distinct differentiation should be made between olfactory and gustatory loss.

The population was characterised by a mean age of $29.82 \pm$ 0.55 years old. It can be stated that in this study, the neurosensory complaints were mainly reported by people of younger age. Existing research also recognizes a significant association between the occurrence of olfactory complaints and people of younger age ${ }^{(7,8)}$. Another important finding in this research was that the prevalence of an olfactory disorder among women was significantly higher than the prevalence among men. A distinct female dominance in the occurrence of neurosensory complaints was also observed in earlier studies ${ }^{(7,12)}$. These observations indicate that the female sex is more prone to neurosensory impairment in comparison to the male sex. As described below, certain selection and reporting biases could explain the dominance of these categories in this study. Though, this trend was also observed in different studies from other countries ${ }^{(7,12)}$. The fact that mainly men from an older age group are affected by a severe form of the disease may explain that the prevalence of these symptoms is higher in women from a younger age group, who more often have a mild form of the disease ${ }^{(16,17)}$.

It is interesting to note that the most prevalent symptoms associated with dysosmia were fatigue, headaches and nasal complaints. This outcome is not in line with the classic presentation of a SARS-CoV-2 infection which is represented by the cardinal symptoms such as dyspnoea, retrosternal pain and fever $(2,3,5)$. A significant difference in duration was observed between the olfactory or gustatory complaints ( $17.95 \pm 0.529$ days) and the associated symptoms. It can be concluded that the duration of the olfactory and/or gustatory impairment is significantly longer than the related symptoms.

Another interesting finding in this study was the strong impact that the affected subjects encountered from these neurosensory complaints. They reported to experience feelings such as annoyance, anger, insecurity and depression and they felt constrained in the performance of various daily activities. Olfactory impairment is known as a invalidating condition and should be acknowledged as an important burden in the life of the affected person ${ }^{(14)}$. Future research should investigate the psychological impact of such disabling complaints in the long term.

In this study, it was established whether the subjects attempted certain treatment options for their olfactory loss. There was also an inquiry into the subjective experienced effect of these interventions. The most favourable outcome was observed in olfactory training, as $95 \%$ of the subjects who started this treatment experienced improvement of their complaints. Given 
the limited number of individuals who have attempted this treatment, these results need to be examined critically. Furthermore, it need to be stressed that spontaneous recovery should be considered when assessing these numbers. Nevertheless, several previous studies have also shown positive results for the use of olfactory training of post-viral origin ${ }^{(9,10)}$. These results further support the idea of olfactory training as effective treatment for this condition ${ }^{(10)}$.

The results from this research suggest that the occurrence of olfactory complaints is of diagnostic significance in the presentation of a SARS-CoV-2 infection, given the observation that $88.2 \%$ of the subjects who got tested in fact were infected with SARS-CoV-2. These observations are in strong agreement with the results obtained from previous studies that have found significant evidence for the association olfactory disorders and the SARS-CoV-2 virus ${ }^{(11,12,18,19)}$. It should be considered that only 93 out of the 500 subjects were qualified to get tested during the acute phase of their illness. Therefore, no conclusions could be made for a large part of the study population. The findings from this study indicate that neurosensory symptoms could be used as diagnostic markers for a SARS-CoV-2 infection and that they should be implemented in the test criteria for a SARS-CoV- 2 infection. These observations are corroborated by several foreign studies where this phenomenon has also been observed and is considered as a strong predictor for a SARS-CoV-2 infection ${ }^{(16,}$ 20, 21).

Certain limitations need to be taken in account when interpreting the results of this research. The following elements could lead to a biased effect on the results of this study. Firstly, online recruitment via social media may have favoured the participation of predominantly the younger age group within our society. Subsequently, it should be noted that the mapping of a subjective complaint as olfactory impairment is challenging. Another important weakness of this study is the fact that only limited subjects were tested during the acute phase of the disease progression, due to the absence of typical COVID-19 symptoms and the limited test capacity at the time. Only $18.6 \%$ of the study population was eligible for a test during the acute course of the disease. Finally, it should be considered that the spontaneous recovery of the complaints is a large confounding factor in the assessment of the effectiveness of the initiated therapies.

\section{Conclusions}

From this research, it can be concluded that olfactory impairment is a prevalent clinical sign of a mild SARS-CoV-2 infection and should be implemented as diagnostic marker in the assessment of COVID-19 disease. The results from this study imply that these neurosensory dysfunctions are particularly manifested in women of younger age. These findings are consistent with several studies in different study populations, which confirms that a SARS-CoV-2 infection in these subcategories is more often characterized by a mild clinical course with neurosensory complaints. The recognition and identification of these complaints as COVID-19 related can allow a more targeted testing in this otherwise asymptomatic patient group, which could be of significant importance in the management of this crisis.

\section{Acknowledgments}

Special thanks to Frederick Acke, Luc Cornette and Hendrik Cornette for critically reading and appraising the manuscript.

\section{Funding}

The authors received no specific funding for this work.

\section{Ethics approval and consent to participate}

The study protocol was approved by The Ethic Committee of Ghent University Hospital. An informed consent was signed by the participants.

\section{Consent for publication}

Not applicable.

\section{Authorship contribution}

MJB: Acquisition of data, Analysis and interpretation of data, drafting of manuscript. SC: Acquisition of data, study conception and design, critical revision of manuscript. Both authors read and approved the final manuscript.

\section{Availability of data and materials}

The datasets used and/or analysed during the current study are available from the corresponding author on reasonable request.

\section{Conflict of interest}

The authors declare that there is no conflict of interest regarding the publication of this article.

\section{References}

1. Harapan $H$, Itoh N, Yufika A, Winardi W Keam S, Te H, et al. Coronavirus disease 2019 (COVID-19): A literature review. J Infect Public Health. 2020;13(5):667-73.

2. Paules $\mathrm{Cl}$, Marston HD, Fauci AS Coronavirus Infections-More Than Just the Common Cold. Jama. 2020;323(8):707-8
3. Pascarella G, Strumia A, Piliego C, Bruno $F$ Del Buono R, Costa F, et al. COVID-19 diagnosis and management: a comprehensive review. J Intern Med. 2020;288(2):192-206.

4. Lee $Y$, Min P, Lee S, Kim SW. Prevalence and Duration of Acute Loss of Smell or Taste in COVID-19 Patients. J Korean Med Sci. 2020;35(18):e174
5. Kim GU, Kim MJ, Ra SH, Lee J, Bae S, Jung J, et al. Clinical characteristics of asymptomatic and symptomatic patients with mild COVID-19. Clin Microbiol Infect. 2020;26(7):948.e1-.e3

6. Whitehead DE, Kelly C, Ahmad N. A case series of patients, including a consultant rhinologist, who all experienced a loss of 
smell associated with confirmed or suspected COVID-19. 2020.

7. Lechien JR, Chiesa-Estomba CM, De Siat $\mathrm{DR}$, Horoi M, Le Bon SD, Rodriguez A, et al. Olfactory and gustatory dysfunctions as a clinical presentation of mild-to-moderate forms of the coronavirus disease (COVID19): a multicenter European study. Eur Arch Otorhinolaryngol. 2020;277(8):2251-61.

8. Kang YJ, Cho JH, Lee MH, Kim YJ, Park CS The diagnostic value of detecting sudden smell loss among asymptomatic COVID19 patients in early stage: The possible early sign of COVID-19. Auris Nasus Larynx. 2020:47(4):565-73.

9. Philpott C. Post-infectious and post-traumatic olfactory loss. Management of Smell and Taste Disorders 2013.

10. Goncalves S, Goldstein BJ. Pathophysiology of Olfactory Disorders and Potential Treatment Strategies. Curr Otorhinolaryngol Rep. 2016;4(2):115-21.

11. Klopfenstein T, Kadiane-Oussou NJ, Toko L, Royer PY, Lepiller Q, Gendrin V, et al. Features of anosmia in COVID-19. Med Mal Infect. 2020;50(5):436-9.

12. Hopkins C, Surda P, Kumar N. Presentation of new onset anosmia during the COVID-19 pandemic. Rhinology. 2020;58(3):295-8.

13. Han AY, Mukdad L, Long JL, Lopez IA Anosmia in COVID-19: Mechanisms and Significance. Chem Senses. 2020.
14. Boesveldt S, Postma EM, Boak D, WelgeLuessen A, Schöpf V, Mainland JD, et al. Anosmia-A Clinical Review. Chem Senses. 2017:42(7):513-23.

15. Qiu C, Cui C, Hautefort C, Haehner A, Zhao J, Yao Q, et al. Olfactory and Gustatory Dysfunction as an Early Identifier of COVID19 in Adults and Children: An International Multicenter Study. Otolaryngol Head Neck Surg. 2020;163(4):714-21.

16. Rocke J, Hopkins C, Philpott C, Kumar N. Is loss of sense of smell a diagnostic marker in COVID-19: A systematic review and metaanalysis. Clin Otolaryngol. 2020;45(6):91422.

17. Vaira LA, Hopkins C, Salzano G, Petrocelli M, Melis A, Cucurullo M, et al. Olfactory and gustatory function impairment in COVID-19 patients: Italian objective multicenter-study. Head Neck. 2020;42(7):1560-1569.

18. Ottaviano G, Carecchio M, Scarpa B, Marchese-Ragona R. Olfactory and rhinological evaluations in SARS-CoV-2 patients complaining of olfactory loss. Rhinology. 2020;58(4):400-401

19. Sedaghat AR, Gengler I, Speth MM. Olfactory Dysfunction: A Highly Prevalent Symptom of COVID-19 With Public Health Significance. Otolaryngol Head Neck Surg. 2020;163(1):12-5.

20. Borsetto D, Hopkins C, Philips V, Obholzer R, Tirelli G, Polesel J, et al. Self-reported altera- tion of sense of smell or taste in patients with COVID-19: a systematic review and meta-analysis on 3563 patients. Rhinology. 2020; 58(5):430-436.

21. Lechien JR, Chiesa-Estomba CM, Place $S$, Van Laethem Y, Cabaraux P, Mat Q, et al. Clinical and epidemiological characteristics of 1,420 European patients with mild-tomoderate coronavirus disease 2019. J Intern Med. 2020; 288(3):335-344.

Sofie Claeys

Department of Head and Skin

Faculty of Medicine and Health

Sciences

University Hospital Ghent

Corneel Heymanslaan 10

9000 Ghent

Belgium

Tel: +32-(0)9332 2332

Fax: +32-(0)9332 4993

E-mail:Sofie.Claeys@uzgent.be

ISSN: 2589-5613 / @2021 The Author(s). This work is licensed under a Creative Commons Attribution 4.0 International License. The images or other third party material in this article are included in the article's Creative Commons license, unless indicated otherwise in the credit line; if the material is not included under the Creative Commons license, users will need to obtain permission from the license holder to reproduce the material. To view a copy of this license, visit http://creativecommons.org/ licenses/by/4.0/ 